\title{
Influence of the Silver Nanocrystal Shape on the Luminous Efficiency of Blue-Emitting Polymer Light-Emitting Diodes
}

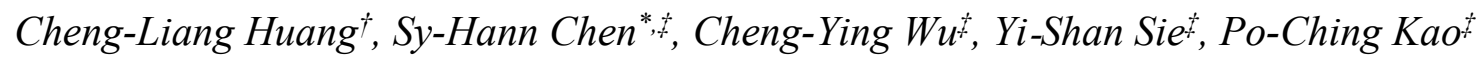

‘Department of Applied Chemistry, National Chiayi University, Chiayi 600, Taiwan

Department of Electrophysics, National Chiayi University, Chiayi 600, Taiwan

*E-mail address: shchen@mail.ncyu.edu.tw 


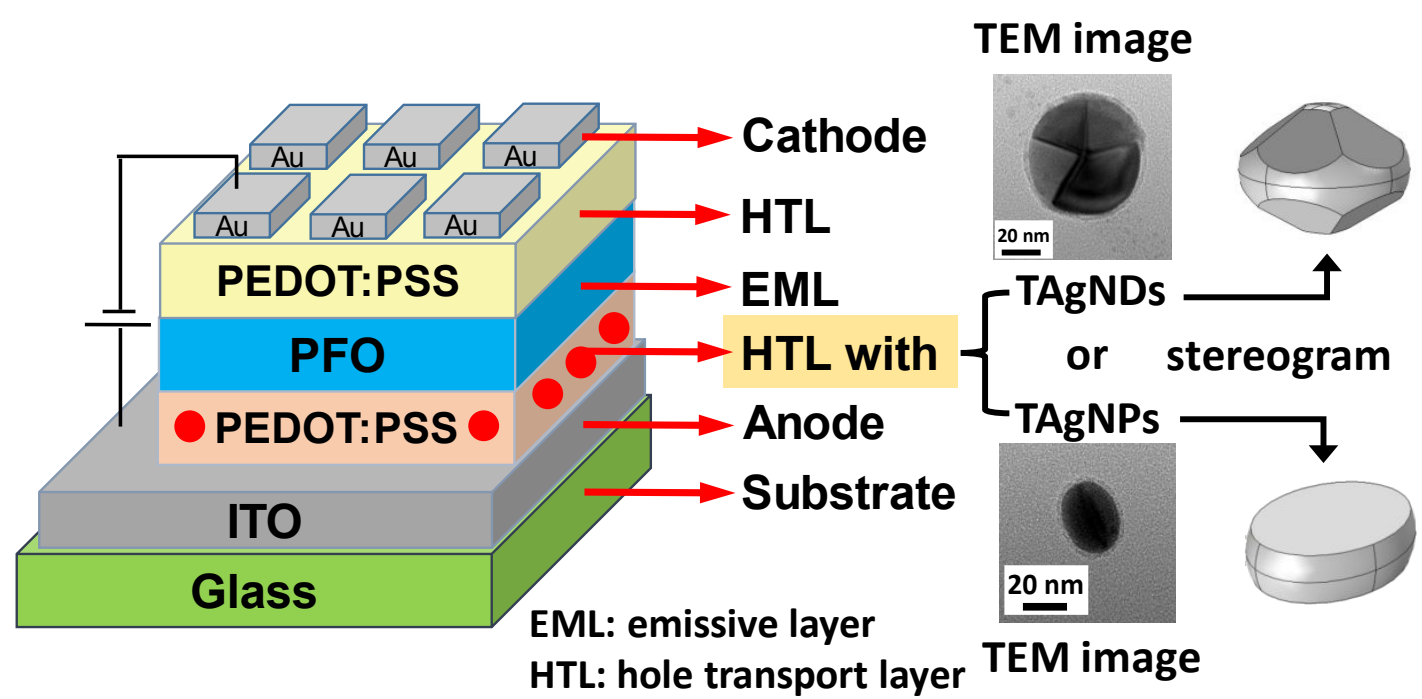

Figure S1. Device architecture of the fabricated hole-only blue-emitting PLEDs with Ag-NCs. 


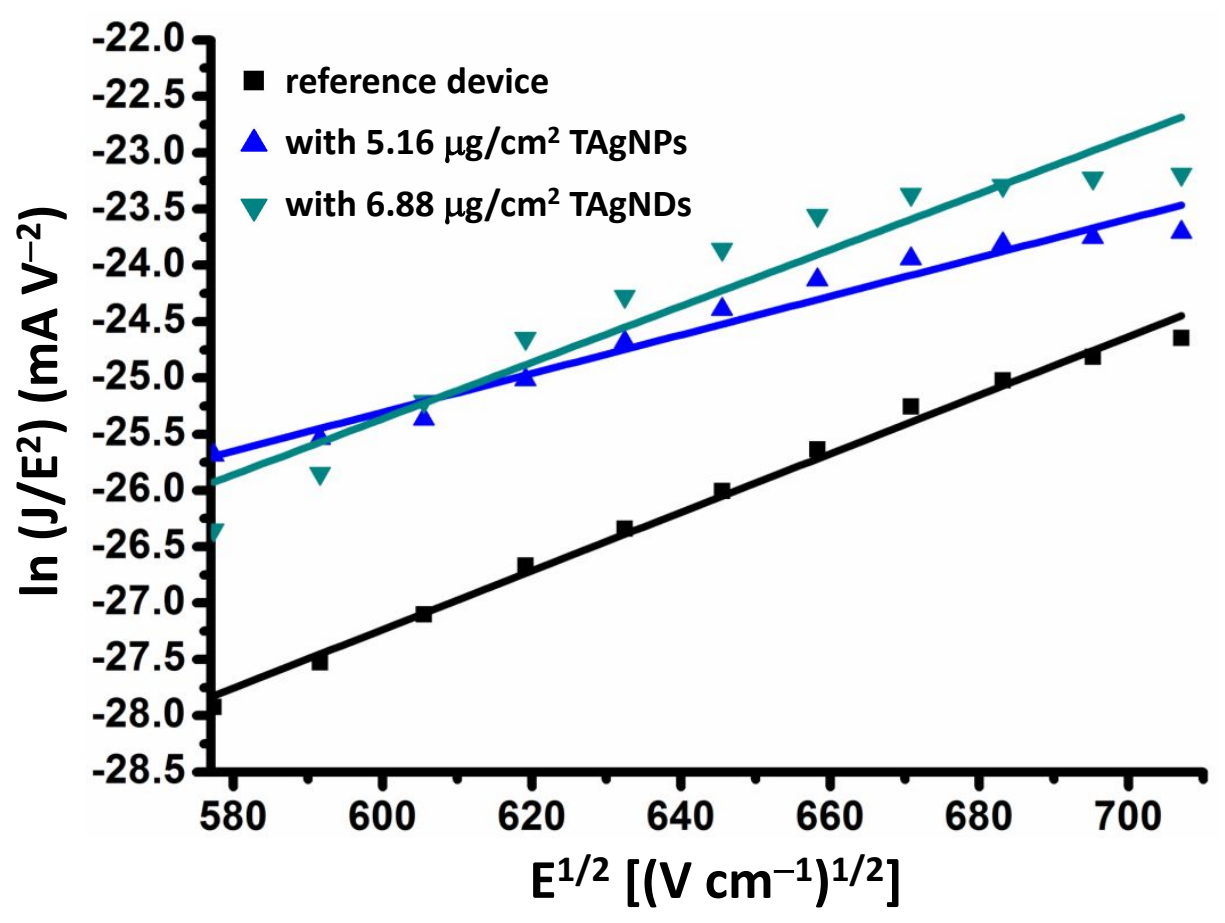

Figure S2. The $\ln \left(\mathrm{J} / \mathrm{E}^{2}\right)-\mathrm{E}^{1 / 2}$ characteristics of the reference and proposed hole-only blue-emitting PLEDs. 


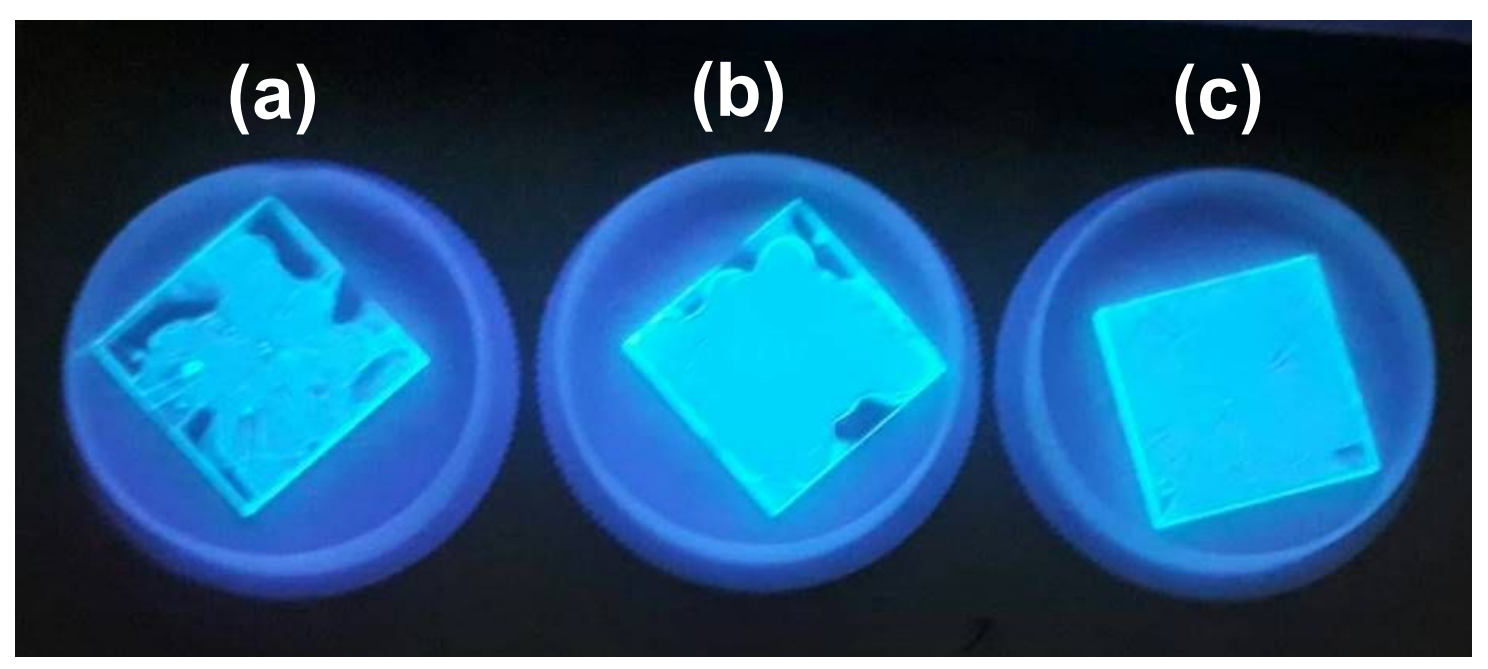

Figure S3. Digital images of the PLEDs under the irradiation of UVA light (365 nm) (a) in the absence and in the presence of (b) TAgNPs and (c) TAgNDs. 

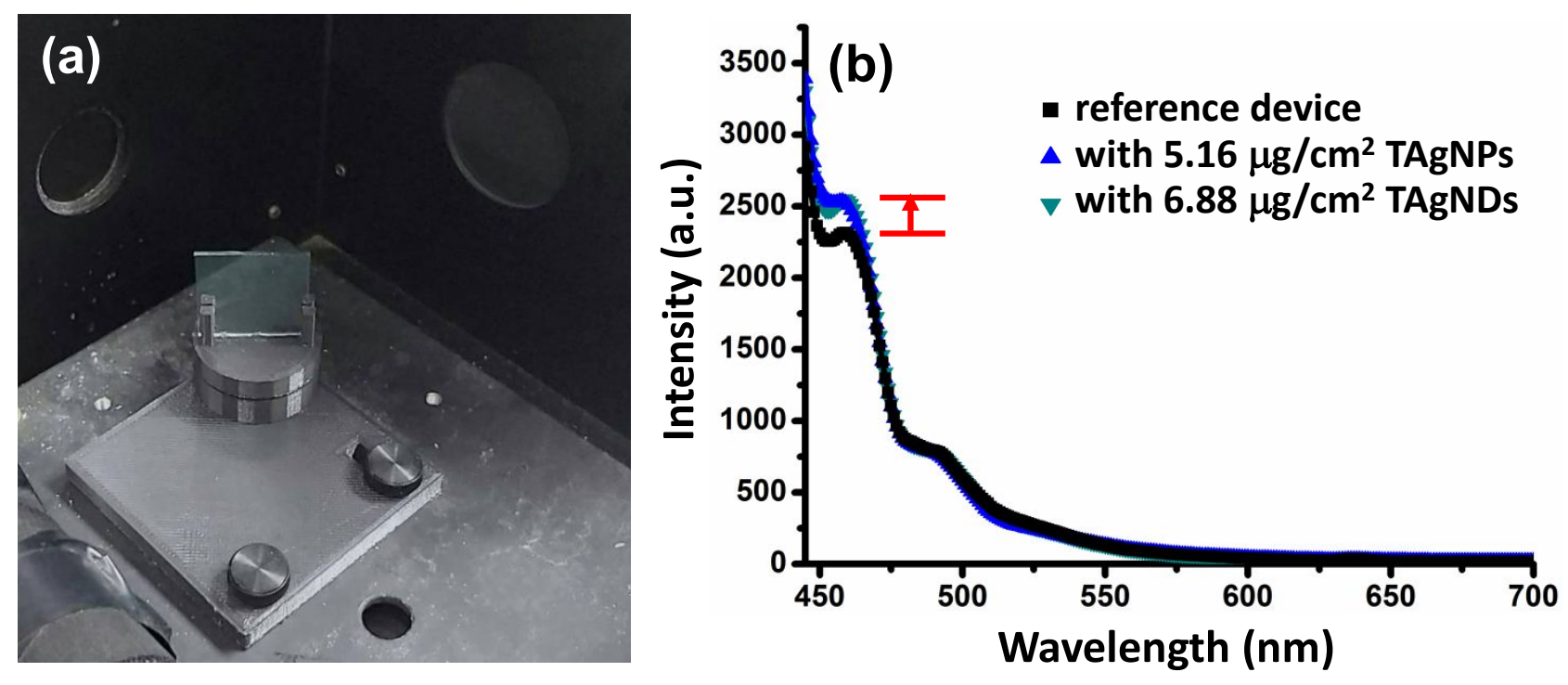

Figure S4. (a) The digital image of the detection geometry inside the fluorescence spectrometer. (b) The PL spectra of the PLEDs. Spectra with black, blue, and green colors denote the PLEDs in the absence of AgNCs, in the presence of TAgNPs and TAgNDs, respectively. Every spectrum was recorded and averaged for three times. The excitation wavelength is $435 \mathrm{~nm}$. 

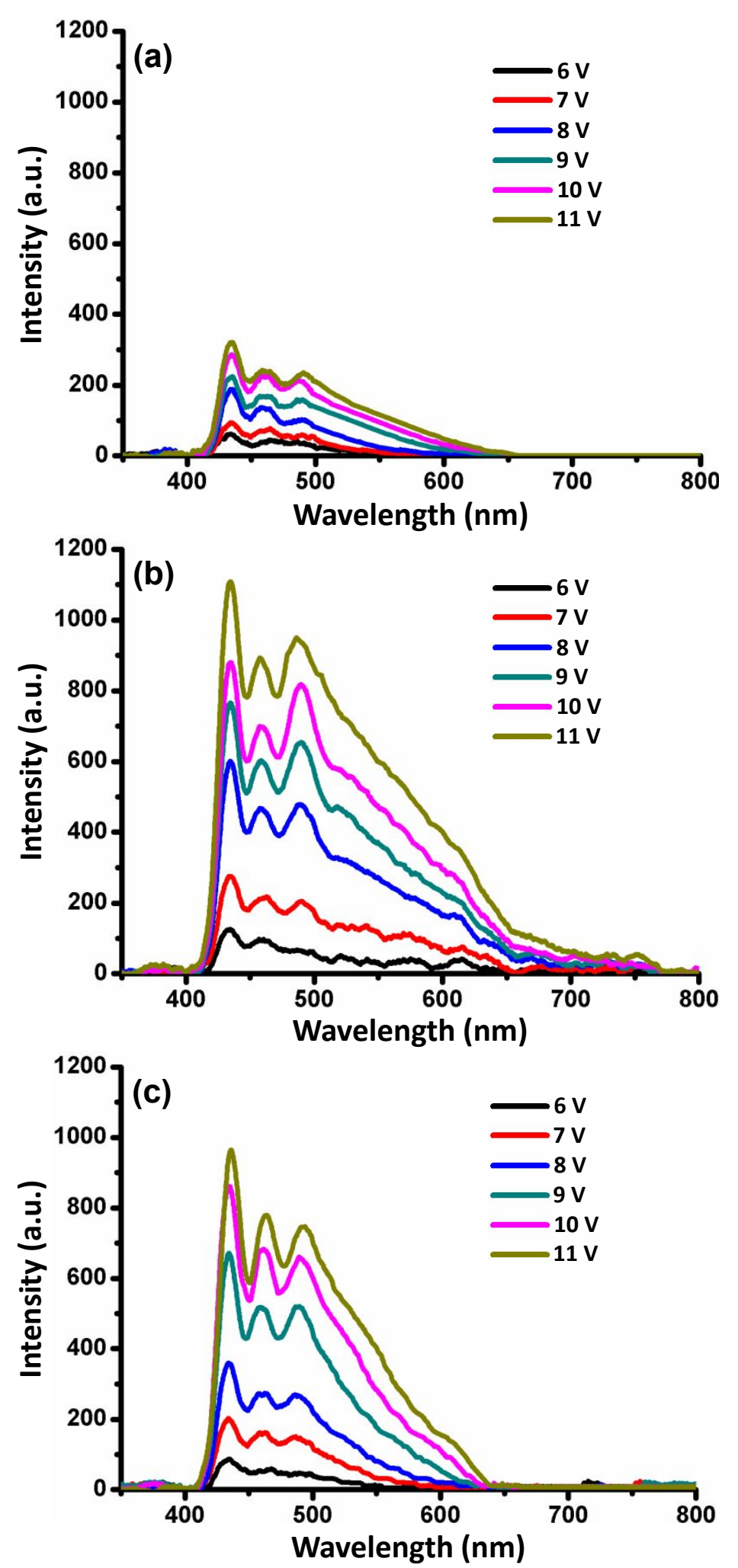

Figure S5. EL spectra of (a) reference blue-emitting PLED, (b) blue-emitting PLED with $6.88 \mu \mathrm{g}$ $\mathrm{cm}^{-2}$ TAgNDs and (c) blue-emitting PLED with $5.16 \mu \mathrm{g} \mathrm{cm}^{-2}$ TAgNPs measured at different bias voltages. 


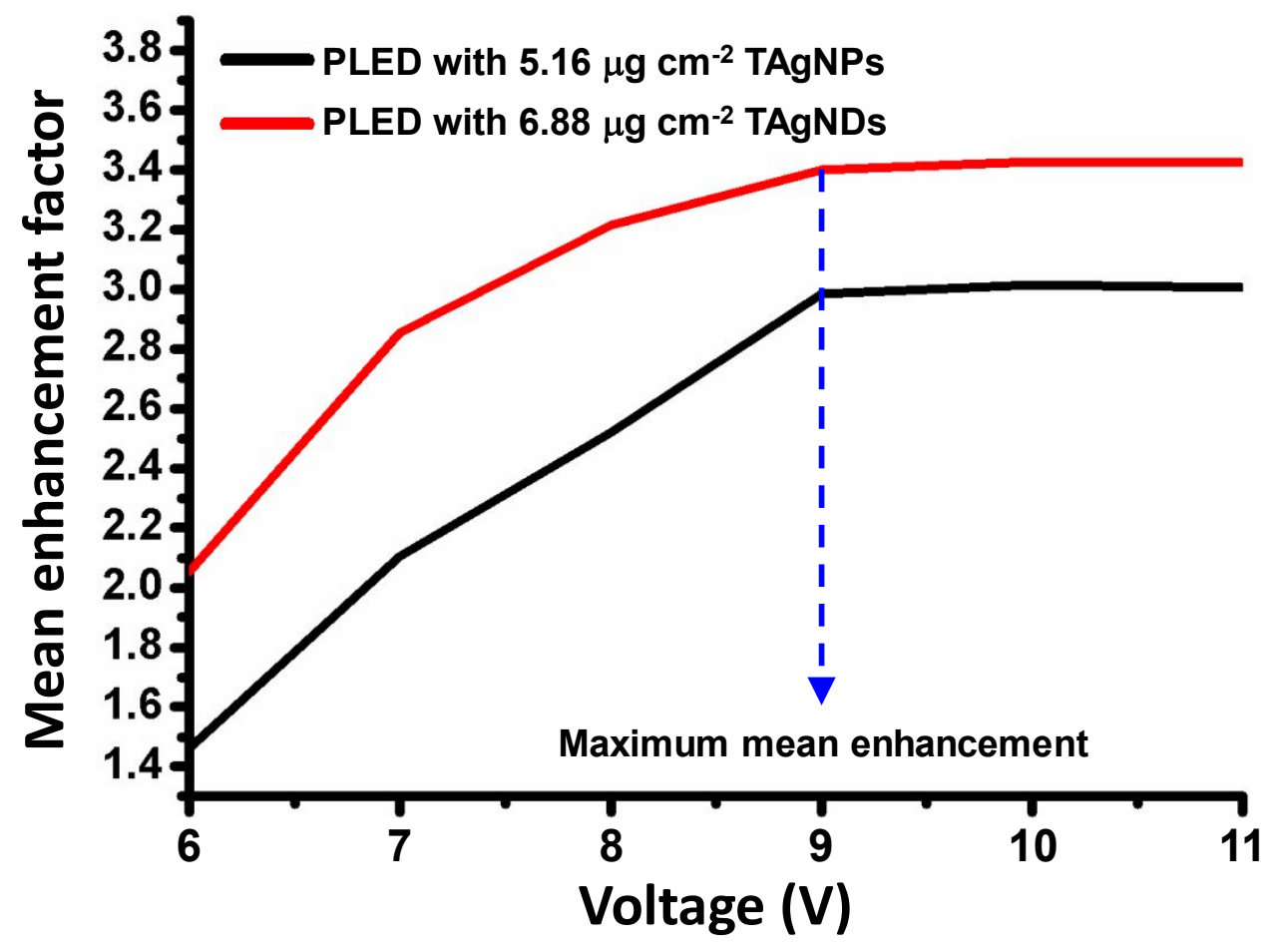

Figure S6. Mean enhancement factor of EL intensity calculated from Fig. S5 as a function of bias voltage. Mean enhancement factor is the average intensity gain between the proposed and reference PLEDs at a wavelength of 430-490 $\mathrm{nm}$. 